\title{
Simulation of JET ITER-Like Wall pulses at high neon seeding rate
}

\author{
G. Telesca ${ }^{1}$, I. Ivanova-Stanik ${ }^{2}$, R. Zagórski ${ }^{2}$, S. Brezinsek ${ }^{3}$, A. Czarnecka ${ }^{2}$, P. \\ Drewelow $^{4}$, C. Giroud ${ }^{5}$, A. Huber ${ }^{3}$, S. Wiesen ${ }^{3}$, M.Wischmeier ${ }^{6}$ and JET contributors* \\ EUROfusion Consortium, JET, Culham Science Centre, Abingdon, OX14 3DB, UK \\ ${ }^{I}$ Department of Applied Physics, Ghent University, B-9000 Gent, Belgium \\ ${ }^{2}$ Institute of Plasma Physics and Laser Microfusion, Warsaw, Poland \\ ${ }^{3}$ Institut fuer Energie-und Klimaforschung-Plasmaphysik Foschungszentrum Juelich GmbH, Juelich, Germany. \\ ${ }^{4}$ Max-Planck Institut fuer Plasmaphysik D-17491 Greifswald, Germany. \\ ${ }^{5}$ CCFE Culham, Abingdon, Oxon. OX14 3DB, UK \\ ${ }^{6}$ Max-Planck Institut fuer Plasmaphysik D-85748, Garching bei Muenchen, Germany.
}

\begin{abstract}
A series of neon seeded JET ELMy H-mode pulses is considered from the modeling as well as from the experimental point of view. For two different $\mathrm{Ne}$ seeding rates and two different D puffing gas levels the heating power, $P_{\text {heat }}$, is in the range 22-29.5 MW. The main focus is on the numerical reconstruction of the total radiated power (which mostly depends on the $\mathrm{W}$ concentration) and its distribution between core and divertor and of Zeff (which mostly depends on the Ne concentration). To model with the self-consistent code COREDIV the core and the SOL two input parameters had to be adjusted case by case: the SOL diffusivity, $\mathrm{D}_{\mathrm{SOL}}$, and the core impurity inward pinch, $v_{\text {pinch }}$. $\mathrm{D}_{\mathrm{SOL}}$ had to be increased with increasing $\Gamma_{\mathrm{Ne}}$ and the level of $v_{\text {pinch }}$ had to be changed, for any given $\Gamma_{\mathrm{Ne}}$, according to the level of $P_{\text {heat }}$ : it decreases with increasing $P_{\text {heat }}$. Since the ELM frequency, $f_{E L M}$, is experimentally correlated with $P_{\text {heat }}$, (it increases with $P_{\text {heat }}$ ) the impurity inward pinch can be seen as to depend on $f_{E L M}$. Therefore, to maintain a low $v_{\text {pinch }}$ level (i.e. high $\left.f_{E L M}\right) \Gamma_{\mathrm{Ne}} / P_{\text {heat }}$ should not exceed a certain threshold, which slightly increases with the $\Gamma_{\mathrm{D}}$ rate. This might lead to a limitation in the viability of reducing the target heat load by Ne seeding at moderate $\Gamma_{\mathrm{D}}$, while keeping $Z_{\text {eff }}$ at acceptably low level. In the considered range of temperatures and densities, the numerical results suggest the balance between friction and thermal forces to be in favour of the frictional drag for tungsten, thus providing a significant $\mathrm{W}$ screening effect.
\end{abstract}

\footnotetext{
* see the Appendix of X. Litaudon et al., Proceedings of the 26th IAEA Fusion Energy Conference 2016.
} 


\section{Introduction}

For a fully metallic device like JET with the ITER-Like-Wall (W divertor, Be wall), made in view to meet the requirements of low fuel retention and low erosion in a next step device, impurity seeding is an essential technique to reduce the power load to the targets, via enhanced edge radiation. Indeed, the naturally occurring radiation losses are low ( $25-30 \%$ of the heating power), as compared to those with carbon target $(\sim 50 \%)$. Significant progress has been made at JET in reducing the heat and particle load to the divertor plates by injecting light impurities as $\mathrm{N}, \mathrm{Ne}$ and Ar, expecially in the ITER-relevant vertical-target configuration (low and high delta), at $\mathrm{I}_{\mathrm{p}}=2.5 \mathrm{MA}$ and $\mathrm{B}_{\mathrm{t}}=2.7 \mathrm{~T}[1,2]$.

In contrast to $\mathrm{Ne}$ injection with carbon PFC, Ne seeding in ILW causes significant changes in the radiation pattern also in the core plasma. Generally, Ne injection not only leads to enahanced radiation in the SOL and around the X-point, as for carbon sorroundings, but also to increased tungsten release, with related enhanced core radiation and, possibly, to reduced ELM frequency. This can cause excessive impurity residence time and may lead to impurity accumulation [3]. This paper, based on experimental JET data as well as on core-SOL selfconsistent modelling, is focusing on the relation between the level of $\mathrm{Ne}$ seeding rate and the change in the impurity transport.

Among a number of experiments carried out recently, a series of Ne seeded low-delta ELMy $\mathrm{H}$-mode pulses with heating power, $P_{\text {heat }}$, up to $29.5 \mathrm{MW}$ and with two different levels of seeding rate as well of gas fueling is considered in the present study.

We have focused our interest on the global radiation properties of these pulses as well as on

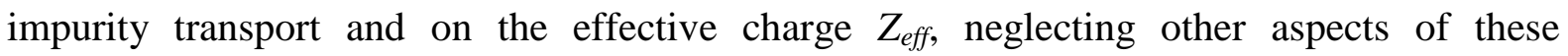
discharges, as, for example, the confinement properties (see ref.[1,2,4]). In particular, an anomalously high $Z_{\text {eff }}$ is observed to occour when, for given levels of $P_{\text {heat }}$ and of gas puffing rate $\Gamma_{\mathrm{D}}$, the Ne seeding exceeds a certain threshold. In this case the incremental $Z_{\text {eff }}$ caused by Ne seeding is higher than that expected from the related incremental radiated power. It is also 
observed that the ELM activity decreases significantly, while the plasma enegy remains unchanged.

In these pulses the volume average density, $\left\langle\mathrm{n}_{\mathrm{e}}\right\rangle$, is in the range $6-7 \times 10^{19} / \mathrm{m}^{3}, P_{\text {heat }}$ from 22 to $29.5 \mathrm{MW}(\mathrm{NBI}+\mathrm{ICRH})$, the two steps in Ne seeding rate are 5 and $12 \times 10^{21} \mathrm{e} / \mathrm{s}$ and the two steps in $\mathrm{D}_{2}$ gas fuelling rate are 1.9 and $3.6 \times 10^{22} / \mathrm{s}$. The radiated power fraction $\left(f_{R A D}=\right.$ $P_{\text {rad }}^{\text {TOT } / ~} P_{\text {heat }}$ ) changes from 0.47 to 0.61 and the ratio of radiated power in the SOL to the total one $\left(P_{r a d}^{S O L} / P_{r a d}^{T O T}\right)$ is between 0.35 and 0.43 . The main aim of this work consists in reproducing numerically, for each pulse, the electron temperature $T_{e}(r)$, and density $n_{e}(r)$ profiles in the core plasma, $P_{r a d}^{T O T}, P_{r a d}^{S O L} / P_{r a d}^{T O T}$ and $Z_{\text {eff }}$ by changing a limited number of inputs : $\left\langle\mathrm{n}_{\mathrm{e}}\right\rangle, P_{\text {heat }}$ and $\Gamma_{\mathrm{Ne}}$ (see next section). It is worth mentioning that for each "shotpoint" the simulation outputs shown in Sect. 3 are obtained from a single run, i.e. all the quantities are calculated simultaneously. Indeed, numerical simulation of these discharges is being made in view of finding the best conditions leading to high $f_{R A D}$ together with acceptable $Z_{\text {eff }}$ for a situation as close as possible to a real experimental pulse.

For the simulations we have used COREDIV code [5], which self-consistently couples the plasma core (1-D) with the plasma edge (2-D) and the main plasma with impurities. Indeed, both the impurity ionization and radiation losses contribute to the establishment of the electon density and temperature profiles. Although the simulations refer to the inter-ELM phase of the discharges, since production as well as flushing out of $\mathrm{W}$ due to ELMs is not accounted for in the present model, the numerical results might be compared with experimental data averaged over several ELM periods [6,7].

Section 2 deals with the description of the numerical model COREDIV. In Sect. 3 the numerical results are compared with experimental data. Discussion is made in Sect.4 and the summary in Sect.4.

\section{The COREDIV code}

Since the energy balance depends strongly on the coupling between the bulk and the scrapeoff layer (SOL) plasma, modeling requires the transport problem to be addressed in both regions simultaneously. The physics model used in the COREDIV code is based on a selfconsistent coupling of the radial transport in the core to the 2D multifluid description of the SOL. 
In the core, given as code input the volume average electron density $\left\langle\mathrm{n}_{\mathrm{e}}\right\rangle$, the $1 \mathrm{D}$ radial transport equations for bulk ions, for each ionization stage of each impurity ion ( $\mathrm{Be}, \mathrm{Ne}$ and W) and for the electron and ion temperature are solved. The electron and ion energy fluxes are defined by the local transport model proposed in ref. [8] which reproduces a prescribed energy confinement law. In particular, the anomalous heat conductivity is given by the expression $\chi_{\mathrm{e}, \mathrm{i}}=\mathrm{C}_{\mathrm{e}, \mathrm{i}} * a^{2} / \tau_{\mathrm{E}} * F(r)$ where $r$ is the radial coordinate, $a$ is the plasma radius, $\tau_{\mathrm{E}}$ is the energy confinement time defined by the ELMy H-mode scaling law and the coefficient $\left(\mathrm{C}_{\mathrm{e}}=\mathrm{C}_{\mathrm{i}}\right)$ is adjusted to have agreement between calculated and experimental confinement times. The parabolic-like profile function $F(r)$, which may slightly change from run to run in order to match with the actual profiles of the experimental pulse to be modelled, can be modified at the plasma edge to provide for a transport barrier of chosen level. The main plasma ion density is given by the solution of the radial diffusion equation with diffusion coefficients $D_{i}=D_{e}=0.2 \chi_{e}$, as in ref.[8]. Note, however, that the solution of the diffusion equation is largely independent of the exact value of $D_{e} / \chi_{e}$. Indeed, a change in $D_{e} / \chi_{e}$ causes a consistent change in the source term, since the average electron density is a COREDIV input. An anomalous pinch velocity can be applied to the main ions to account for possible density peaking. In all these simulations $v_{\text {pinch }}($ main) $=-0.05 \mathrm{~m} / \mathrm{s}$. For the auxiliary heating, parabolic-like deposition profile is assumed $P_{\text {aux }}(r)=P_{0}\left(1-r^{2} / a^{2}\right)^{y}$ where $y$ is in the range 1.53 , depending on the quality of the auxiliary heating, NBI or/and ICRF. For all the pulses considered in this study $y=2$, since the fraction of ICRH power level to the total power is nearly constant, $\mathrm{P}_{\mathrm{ICRH}} / \mathrm{P}_{\mathrm{NBI}}=0.2$. The impurity diffusion coefficient is set to be equal to that of the main ions and an anomalous impurity pinch is given as input, in the range 0 to $-1 \mathrm{~m} / \mathrm{s}$ for the pulses here considered (see Sect.3). The pinch profile increases linearly, in absolute value, from zero at the plasma centre to its maximum (the value we quote) at $r=a$.

In the SOL the 2D boundary layer code EPIT [9] is used, which is primarily based on Braginskii-like equations for the background plasma and on rate equations for each ionization stage of each impurity species. An analytical description of the neutrals is used, based on a simple diffusive model. COREDIV takes into account the plasma and seeded impurities recycling in the divertor as well as the sputtering processes at the target plates including $\mathrm{W}$ sputtering by deuterons, self-sputtering and sputtering due to seeded impurities. (For deuterium and neon sputtering and tungsten self-sputtering the yields given in refs. $[\mathbf{1 0 , 1 1 ]}$ 
are used). W self-sputtering accounts for about $10 \%$ of the total $\mathrm{W}$ release, for the simulations presented in this paper. The recycling coefficient is an external parameter which in COREDIV depends on the level of the electron density at the separatrix, $\mathrm{n}_{\mathrm{e}_{-} \text {sep }}$, given as an input, and increases with increasing $\mathrm{n}_{\mathrm{e}_{-} s e p}$.

A simple slab geometry (poloidal and radial directions) with classical parallel transport and anomalous radial transport $\left(D_{S O L}=\chi_{i}=0.5 \chi_{e}\right.$, where $\chi_{e}$ ranges typically $\left.0.5-1.5 \mathrm{~m}^{2} / \mathrm{s}\right)$, is used and the impurity fluxes and radiation losses by impurity ions are calculated fully selfconsistently. Although the values of the transport coefficients in the SOL are generally quite comparable to those at the separatrix, in the present simulations the value of $D_{S O L}$ is set arbitrarily (in the range $0.4-0.7 \mathrm{~m}^{2} / \mathrm{s}$ ) in order to match with the core-SOL distribution of the radiated power, depending on the different levels of Ne seeding rate (see next section). All the equations are solved only from the midplane to the divertor plate, assuming inner-outer symmetry of the problem. This implies that the experimental in-out asymmetries, observed especially at high density-high radiation level, are not reproduced in COREDIV results. However, for all the different situations examined so far (with carbon plates and with the ILW, and with different seeding levels $[6,12]$ ) the COREDIV numerical reconstructed total radiation in the SOL matches well with the total experimentally measured SOL radiation, indicating that for JET conditions the edge-core COREDIV model can describe the global trend of this important quantity.

The coupling between the core and the SOL is made by imposing continuity of energy and particle fluxes as well as of particle densities and temperatures at the separatrix. The computed fluxes from the core are used as boundary condition for the SOL plasma. In turn, the values of temperatures and of densities calculated in the SOL are used as boundary conditions for the core module

\section{Experiments and simulations}

Fig. 1 shows some time-dependent traces of the four pulses under exam. In JPN 87190 (top left) $P_{\text {heat }}(\mathrm{NBI}+\mathrm{RF})$ is $22 \mathrm{MW}, \Gamma_{\mathrm{Ne}}=0.510^{22} \mathrm{e} / \mathrm{s}$ and for $\mathrm{t}<14.5 \mathrm{~s}$ with $\Gamma_{\mathrm{D}}=3.6 \times 10^{22} \mathrm{e} / \mathrm{s}$ ELM activity is stationary with $f_{E L M}=40 \mathrm{~Hz}, Z_{\text {eff }}=1.9$ and $f_{R A D}=0.47$. Starting from $\mathrm{t}=14.5 \mathrm{~s}$ the gas fueling decreses to $\Gamma_{\mathrm{D}}=1.9 \times 10^{22} \mathrm{e} / \mathrm{s}$ and the ELM behavior is non-stable, alternating periods of ELM activity with $f_{E L M}=40 \mathrm{~Hz}$ with ELMy-free periods. Correlated also with a 
slight decrease in $\left\langle\mathrm{n}_{\mathrm{e}}\right\rangle, Z_{\text {eff }}$ goes up to 2.4 and $f_{R A D}=0.52$. In JPN 87191 (top right) the two gas fuelling steps are interchanged in time, but their values are quite similar to those in the previous pulse, while the neon seeding level remains $\Gamma_{\mathrm{Ne}}=0.510^{22} \mathrm{e} / \mathrm{s}$. With $P_{\text {heat }}=26 \mathrm{MW}$, ELM activity is stable at both $\Gamma_{\mathrm{D}}, f_{E L M}=60-70 \mathrm{~Hz}$, with $Z_{\text {eff }}$ around 2 and $f_{R A D}=0.54$ and 0.47 respectively. In JPN 87192 (bottom left), $\Gamma_{\mathrm{Ne}}=1.210^{22} \mathrm{e} / \mathrm{s}$ while all the other inputs are keept as in the previous pulse. ELM activity is non-stable with $f_{E L M}=5-9 \mathrm{~Hz}, Z_{\text {eff }}$ in the range 3.43.0 and $f_{R A D}$ around 0.55. Adding $1 \mathrm{MW}$ of ICRH, as in JPN 87194 (bottom right) up to $\mathrm{t}=14.5 \mathrm{~s}$, does not improve the situation with respect to $Z_{\text {eff }}$ and $f_{E L M}$. At $\mathrm{t}=14.5 \mathrm{~s}, 2$ more megawatts NBI are added resulting in $P_{\text {heat }}=29.5 \mathrm{MW}$. Even though no significant

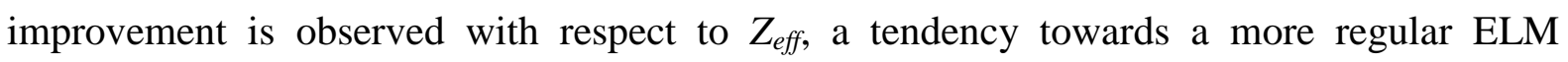
behavior might be seen, with $f_{E L M}$ which becomes $15 \mathrm{~Hz}$. A summary of the experimental data for the 7 time slices ("shot-points") belonging to the 4 pulses examined is given in table I. In the following, comparison is made between the experimental data of table I and COREDIV numerical results.

Before comparing and discussing the global quantilties of the core and the SOL, the experimental (from High Resolution Thomson Scattering diagnostic) and simulated $T_{e}$ and $n_{e}$ profiles in the core plasma of one of the four pulses here considered (JPN $87194 \mathrm{t}=14.9 \mathrm{~s}$ ) are shown in Fig. 2, as an example. Although some discrepancies experiment-simulation can be seen, these discrepancies are marginally influent for the numerical reconstruction of the global quantities of the core plasma. Indeed, due to the rather flatness of the $n_{e}$ profile, small differences experiment-simulation in the $T_{e}$ profile in the confined plasma cause a marginal effect on the level of the reconstructed radiated power. On the other hand, recalling that impurity transport is set to be anomalous in the present version of COREDIV (see Sect.2), the differences experiment-simulation in the $\mathrm{T}_{\mathrm{e}}$ gradients are also of minor concern.

Fig.3a shows the tomographic reconstruction from bolometric data for one typical $\mathrm{Ne}$ seeded JET pulse. It is apparent that a clear limit between the radiation emitted in the core and that in the SOL is difficult to define within the uncertainties in space and absolute numbers of the measurement, especially in the vicinity of the $\mathrm{x}$-point. Therefore, we have followed a procedure, used at JET [13], by which all the power radiated below $Z=-1 \mathrm{~m}$ is considered as divertor radiation. Since neon radiates partly in the SOL and partly at the very edge of the plasma core around the $\mathrm{X}$-point, this assumption turns out to include the total radiation 
emitted by $\mathrm{Ne}$ in what we call "experimental divertor radiation". Being the core module of COREDIV one-dimensional (see above), the simulated Ne radiation inside the separatrix is poloidally uniformly distributed and it is located, as in the experiment, at the very edge of the core (see Fig. 3b). To compare consistently simulations with measurements we have therefore added the COREDIV neon radiation emitted at the very edge of the core to the COREDIV SOL radiation, resulting in the "simulated divertor radiation". In Fig. 4 the seven "shotpoints" have been ordered according to $P_{\text {heat }}$ and the four points referring to $P_{\text {heal }}=26 \mathrm{MW}$ have been slithly displaced in $P_{\text {heat }}$ to avoid superposition. The points referring to $\Gamma_{\mathrm{Ne}}=1.2$ $10^{22} \mathrm{e} / \mathrm{s}$ have been drawn with larger symbols. Figs.4a,b,c show that $P_{\mathrm{rad}}^{\mathrm{TOT}}, P_{\mathrm{rad}}^{\mathrm{SOL}} / P_{\mathrm{rad}}^{\mathrm{TOT}}$ and $Z_{\text {eff }}$ have been numerically reconstructed with sufficient accuracy. Fig.4d shows that the experimental $f_{R A D}$ (as well as the simulated one) does not increase significantly by increasing $\Gamma_{\mathrm{Ne}}$, which, however, leads to a clear increase in $Z_{\text {eff. }}$ This point is made more clear by noting that the quantity $P_{\mathrm{rad}}^{T O T} /\left(\right.$ Zeff-1) $\mathrm{x} \mathrm{\textrm {n } _ { \mathrm { e } } ^ { 2 }}$ [14] drops nearly by a factor of 2 at high $\Gamma_{\mathrm{Ne}}$ (Fig.4e). Figs. 4f and 4g show that the volume averaged $\mathrm{W}$ concentration (from SXR) and the central plasma $\mathrm{Ne}^{10+}$ ion concentration (from CXRS) match relatively well with the reconstructed ones, considering the error bar (over 20\%) of the measurements. The electron temperature at the outer stike point, measured by LP (with significant error bar), is in the range 6-9 eV (9 eV for the pulse at $26 \mathrm{MW}$ with $\left.\Gamma_{\mathrm{Ne}}=0.5 \times 10^{22} \mathrm{e} / \mathrm{s}\right)$, while the simulated one is in the range 4-6.5 eV. Considering that the experimental $\mathrm{T}_{\mathrm{e}}$ at the inner strike point is about 2-3 eV lower than at the outer one, the discrepancy experiment-simulation is consistent with the COREDIV SOL model, in which in-out symmetry is assumed (only one target plate, see Sect. 2). Fig.4h shows that high $\Gamma_{\mathrm{Ne}}$ level is an effective tool to reduce the power to the plate also at high $P_{\text {heat }}$. (Note that in Fig. $4 \mathrm{~h} \mathrm{P}_{\mathrm{SOL}}$ is calculated in COREDIV summing up all the losses inside the separatrix, including the Ne radiation in the confined plasma). The simulated recycling D fluxes, $\Gamma_{\mathrm{D}}$, are about a factor of 2 higher than the LP experimental ones. This depends on the value of the COREDIV input electron density at the separatrix, $\mathrm{n}_{\mathrm{e} \_ \text {sep. }}$. Indeed, the recycling deuterium flux is not a free parameter in the model (see Sect.2), but it is linked to the input value of $\mathrm{n}_{\mathrm{e}_{\_} \text {sep }}$, which, in all these simulations is in the range 0.45$0.5 \mathrm{x}<\mathrm{n}_{\mathrm{e}}>$. [6]

Together with $P_{\text {heat }},\left\langle\mathrm{n}_{\mathrm{e}}\right\rangle$ and $\Gamma_{\mathrm{Ne}}$ two other input parameters have been changed "shot-point" to "shot-point" in order to match simulations with experiments: $\mathrm{D}_{\mathrm{SOL}}$ and the anomalous 
impurity pinch, $v_{\text {pinch }}$. Differently from modelling of unseeded pulses with $\mathrm{D}_{\mathrm{SOL}}=0.25 \mathrm{~m}^{2} / \mathrm{s}$, it has been set $D_{\text {SOL }}=0.45 \mathrm{~m}^{2} / \mathrm{s}$ for the points at $\Gamma_{\mathrm{Ne}}=0.5 \times 10^{22} \mathrm{e} / \mathrm{s}$ and $D_{\mathrm{SOL}}$ up to $0.65 \mathrm{~m}^{2} / \mathrm{s}$ for the points at $\Gamma_{\mathrm{Ne}}=1.2 \times 10^{22} \mathrm{e} / \mathrm{s}$. This dependence of $\mathrm{D}_{\mathrm{SOL}}$ on the level of $\Gamma_{\mathrm{Ne}}$ is made to match the simulated with the experimental $P_{\mathrm{rad}}^{S O L}$. To match both the simulated $P_{\text {rad }}^{\text {core }}$ (i.e. $P_{\mathrm{rad}}^{\text {TOT }}$ - $P_{\text {rad }}^{S O L}$ ) and $Z_{\text {eff }}$ with the experimental data, $v_{\text {pinch }}$ had to be changed from $-0.3 \mathrm{~m} / \mathrm{s}$ up to $0.8 \mathrm{~m} / \mathrm{s}$. It might be observed (see Fig. $4 \mathrm{f}$ ) that, in contrast to $\mathrm{c}_{\mathrm{Ne}}, \mathrm{c}_{\mathrm{W}}$ is relatively insensitive to the application of a high $v_{\text {pinch }}$ for the pulses at higher $\Gamma_{\mathrm{Ne}}$. This does not depend on a reduced W flux at higher $\Gamma_{\mathrm{Ne}}$ (see Fig.5), but on the parallel transport in the SOL. Indeed, from the modelling point of view, the ratio between the impurity density in the central plasma and that at the separatrix is identical for $\mathrm{Ne}$ and $\mathrm{W}$ (i.e. the effect of the pinch is the same for both impurities). What changes is the ratio between the density at the separatrix and the flux at the plate, which is higher for $\mathrm{Ne}$ than for $\mathrm{W}$ up to a factor of 4 . This numerical result, supported by experimental data, suggests that the balance between friction and thermal forces in the $\mathrm{SOL}$ is in favour of the frictional drag more for $\mathrm{W}$ than for $\mathrm{Ne}[15]$, providing for $\mathrm{W}$ a high divertor retention and screening effect.

It turns out that $v_{\text {pinch }}$ is correlated with $f_{E L M}$, as seen in Fig. 6a. It has to be pointed out, however, that there is no experimental evidence of change in impurity density peaking with decreasing $f_{E L M}$ and that the $\mathrm{T}_{\mathrm{e}}$ profile is centrally peaked for all these (partly) RF centrally heated plasmas, while the $\mathrm{n}_{\mathrm{e}}$ profile is flat. Considering, moreover, that $v_{\text {pinch }}$ is a numerical tool introduced to change the impurity dwell time, a different numerical approach has been sought to account for the increased $Z_{\text {eff }}$ at low $f_{E L M}$. In fact, a new set of simulations has been performed in which the impurity inward pinch is keept constant $\left(v_{\text {pinch }}=-0.3 \mathrm{~m} / \mathrm{s}\right)$ for all the seven shot points and machting between experiments and simulations is searched by adjusting the impurity diffusion coefficient in the core plasma, $D_{\text {imp-core }}$. In all the simulations previously reported the impurity diffusivity in the core plasma is set to be equal to that of the main ions, $D_{\text {imp-core }}=\mathrm{D}_{\text {main ions }}$ (see Sect. 2), while in the new set of simulations $D_{\text {imp-core }} / \mathrm{D}_{\text {main- }}$ ions has been scanned to find the best match experiments-simulations. The numerical results previously reported, as in Fig. 4, have been fairly well reproduced applying values of $D_{\text {imp- }}$ ${ }_{c o r} / \mathrm{D}_{\text {main ions, }}$, which decrase with decreasing $f_{E L M}$, Fig, 6b. Recalling that in the COREDIV model $D_{\text {main ions }}=D_{e}=0.2 \chi_{e}$ the decrease in $D_{\text {imp-core }} / D_{\text {main ions }}$ implies an increase in $\tau_{\text {imp }} / \tau_{\mathrm{E}}$, where $\tau_{\text {imp }}$ is the impurity dwell time. 
With respect to the mechanism responsible for the reduced $f_{E L M}$ at higher Ne seeding rate it seems reasonable to relate the drop in $f_{E L M}$ with the little increase in the radiated power from the main plasma volume. With larger core radiation, more time is needed to restore the pedestal with the heat flow from the plasma core after an ELM crash [16]. However, considering also the modest reduction in the power to the SOL for the three "shot points" at the higher $\mathrm{Ne}$ seeding rate and the enhanced radiation around the $\mathrm{X}$-point, other driving mechanisms cannot be excluded.

Since the key parameters of this study are $\Gamma_{\mathrm{Ne}}, P_{\text {heat }}$ and $\Gamma_{\mathrm{D}}$, the seven data points above considered plus two from JPN 87194 at $\mathrm{t}<14 \mathrm{~s}$ and three points belonging to a quite similar series with $P_{\text {heat }}=24 \mathrm{MW}$ and $\Gamma_{\mathrm{D}}=4.1 \times 10^{22} \mathrm{el} / \mathrm{s}$ (JPN 87090-92 [7]) have been labelled according to their value of $\Gamma_{\mathrm{Ne}} / P_{\text {heat }}$ and plotted as function of $\Gamma_{\mathrm{D}}$, Fig 7. Although it is not possible to draw any quantitative dependence from these data, the plot shows the positive effect of higher gas puffing rate on enhancing the level of $\Gamma_{\mathrm{Ne}} / P_{\text {heat }}$ at which $f_{E L M}$ remains in excess of $40 \mathrm{~Hz}$.

\section{Discussion}

The absolute value of the numerical neon seeding rate, $\Gamma_{\mathrm{Ne}}($ num), used in COREDIV to match simulation-experiment for the four "shot points" at $\Gamma_{\mathrm{Ne}}=0.5 \times 10^{22} \mathrm{e} / \mathrm{s}$ is lower than the experimental one. The input $\Gamma_{\mathrm{Ne}}($ num) depends, indeed, on the input $\mathrm{Ne}$ recycling coefficient, $\mathrm{R}_{\mathrm{Ne}}$, as shown in Fig 8 for one of the considered pulses at $\Gamma_{\mathrm{Ne}}=0.5 \times 10^{22} \mathrm{e} / \mathrm{s}$. Since we made, arbitrarily, the choice of setting $R_{\mathrm{Ne}}=0.8$, the resulting numerical Ne seeding rate is $\Gamma_{\mathrm{Ne}}=0.32 \times 10^{22} \mathrm{e} / \mathrm{s}$ i.e. about $0.65 \times \Gamma_{\mathrm{Ne}}$ (experimental).

More relevant, it was not possible to model satisfactorily the three " shot points" at higher $\Gamma_{\mathrm{Ne}}$ by increasing in COREDIV the $\Gamma_{\mathrm{Ne}}$ by a factor of 2.4 with respect to that of the four "shot points" at lower $\Gamma_{\mathrm{Ne}}$, as in the experimental $\mathrm{Ne}$ inlet valve signals (see Fig. 1). Match of $P_{\text {rad }}^{T O T}$ and of its distribution between core and SOL, of $Z_{\text {eff }}$ and of the impurity concentrations was only possible by increasing $\Gamma_{\mathrm{Ne}}$ by a factor of $1.45-1.5$, and by changing $\mathrm{D}_{\text {imp }}$ and $v_{\text {pinch }}$, as mentioned in Sect.3. For example (see Fig. 9), starting from the shot point 87191 at $\mathrm{t}=14.5$ (experimental $\Gamma_{\mathrm{Ne}}=0.5 \times 10^{22} \mathrm{e} / \mathrm{s}$ ) and going toward the shot point 87192 at $\mathrm{t}=14.5\left(P_{\mathrm{rad}}^{T O T}=15.2, P_{\mathrm{rad}}^{S O L} / P_{\mathrm{rad}}^{T O T}=0.4, Z_{e f f}=3\right.$, experimental $\left.\Gamma_{\mathrm{Ne}}=1.2 \times 10^{22} \mathrm{e} / \mathrm{s}\right)$, 
increasing $\Gamma_{\mathrm{Ne}}$ (numerical) by a factor 1.6 the resulting $P_{\mathrm{rad}}^{T O T}(=15.6 \mathrm{MW})$ and $P_{\text {rad }}^{S O L} / P_{\text {rad }}^{\text {TOT }}(=0.41)$ reproduce almost satisfactorily the experimental values of the shot point $87192 \mathrm{t}=14.5 \mathrm{~s}$, but $Z_{\text {eff }}(=2.34)$ as well as the impurity concentrations are too low. Increasing $\Gamma_{\mathrm{Ne}}($ num $)$ by a factor $1.9, P_{\mathrm{rad}}^{T O T}(=16.1 \mathrm{MW})$ and $P_{\mathrm{rad}}{ }^{S O L} / P_{\mathrm{rad}}{ }^{T O T}(=0.43)$ are somewhat higher that the experimental values while $Z_{\text {eff }}(=2.6)$ as well as the impurity concentrations remain lower. Incresing further $\Gamma_{\mathrm{Ne}}(\mathrm{num})$ by a factor 2.3 , the resulting numerical $P_{r a d}^{T O T}(=16.8 \mathrm{MW})$ and $P_{\mathrm{rad}}{ }^{S O L} / P_{\mathrm{rad}}{ }^{T O T}(=0.47)$ are quite above the experimental values while $Z_{\text {eff }}(=2.87)$ and the impurity concentrations are still a little lower than in the experiment.

The reason for this discrepancy is not clear, so far. Among many concurrent possibilities, this might be linked to the over-simplified COREDIV geometry of the SOL, which does not account for the divertor closure of the vertical target configuration of these pulses, with possible related changes in the neon divertor compression. Indeed, in the case of impurity recycling in the divertor (rare gases) impurity "compression" by the frictional drag may occur [17]. (In JET the neutral Ne pressure in the divertor is not known, which renders not possible an estimate of the pumping efficiency). Also the experimental strong increase in the radiation from around the X-point may play a role. The reduction of $\mathrm{T}_{\mathrm{e}}(\mathrm{X}$-point) leads to a shift in the ionization and recombination front [18], with possible related change of the neutral compression in the divertor. Another possibility is related to the fact that while in COREDIV the sedeed neon enters directly the plasma, in the experiment neon is puffed in the private region: increasing the divertor density with increasing $\Gamma_{\mathrm{Ne}}(\exp )$, the $\mathrm{Ne}$ penetration depth may decrease, resulting in the reduction of the effective Ne seeding rate [19].

Using the actual steady-state version of COREDIV it is not possible to simulate the effect on $\mathrm{W}$ release caused by single ELMs. However, for $\mathrm{T}_{\mathrm{e} \_\mathrm{pl}}$ above 3-4 eV the $\mathrm{W}$ sputtering yield by $\mathrm{Ne}$ together with that by Be and by $\mathrm{W}$ self-sputtering provide a simulated $\mathrm{W}$ flux, which is comparable to that experimentally observed for the most common JET ELMy H-mode situations, once the data are averaged over times $\tau_{\mathrm{A}} \gg>1 / f_{E L M}[6]$. Indeed, in JET ELMy-H mode the experimental $\Gamma_{\mathrm{W}}$ is found to be in the range $3-9 \times 10^{19} / \mathrm{s}[20,21]$, as in COREDIV simulations. In particular, for this series of pulses the intensity of the WI emission line at 401 $\mathrm{nm}$, measured at the outer divertor, shows a slight increase with increasing $\Gamma_{\mathrm{Ne}}$, in 
qualitative agreement with the calculated COREDIV total $\mathrm{W}$ fluxes, which increase, in total, from about 6 to $9.5 \times 10^{19} / \mathrm{s}$, Fig.5.

The necessity of introducing in the simulations an anomalous inward pinch (or an "ad hoc" decrease in the impurity diffusion coefficient) to match experiment-simulation for the pulses at low $f_{E L M}$ is correlated with the impurity flushing out mechanisms by ELMs [16], and, specifically, with the experimentally observed decrease in $\mathrm{W}$ density in the core plasma for $f_{E L M}$ above $40 \mathrm{~Hz}$ [21]. In fact, ELMs are simultaneously the main cause of $\mathrm{W}$ release and of impuritiy outflow [22].

Taking $40 \mathrm{~Hz}$ as the minimal $f_{E L M}$ for impurity accumulation avoidance and considering that for these JET pulses $\tau_{\mathrm{E}}$ is $0.15-0.2 \mathrm{~s}$, the resulting $\tau_{\mathrm{E}} \times f_{E L M}$ is $6-8$, which is not far from the value of 10 , found in previous studies as the minimal value of $\tau_{\mathrm{E}} \mathrm{x} f_{E L M}$ to avoid $\mathrm{W}$ accumulation [22,23].

\section{Summary and conclusions}

In spite of the inner-outer symmetry assumption and of the analytical description of the neutrals which both might hamper the validity of the SOL model especially for situations close to detachment, the global properties of the SOL, as the radiated power and the average electron temperature at the plate, are sufficiently well numerically reproduced, due also to the adjustement of the particle diffusion coefficient in the SOL, $\mathrm{D}_{\mathrm{SOL}}$. The change in $\mathrm{D}_{\mathrm{SOL}}$ is a technical tool to fitting the numerical $P_{r a d}^{S O L}$ with the experimental one and although it reflects the increase in collisionality with decreasing the divertor temperature it includes also the action of other mechanisms as change in frictional forces and in flux expansion [1]. An inward impurity pinch (or, alternatively, a reduction in the impurity diffusivity) had to be introduced in order to model the pulses at high neon seeding rate, which disply abnormally high $Z_{\text {eff }}$ and Ne concentration. This is experimentally related to the decrease in $f_{E L M}$, likely dependent on the reduction of the power to the SOL.

COREDIV results show that the frictional drag is the main parallel transport mechanism for $\mathrm{W}$ in the SOL, thus providing a significant $\mathrm{W}$ screening.

The reason why the numerical $\mathrm{Ne}$ seeding rate is not linearly related to the experimental one remains an open question, at present. Analysis with more sophisticated models and/or more experimental data would be needed to clarify this point. 
Experiments and COREDIV modelling indicate that Ne seeding is an efficient method to maintain the power load to the target plates at acceptable level also at high $P_{\text {heat }}$ (order 30 MW) in JET-ILW pulses. However, the level of $\Gamma_{\mathrm{Ne}}$ should be modulated according to the level of $\Gamma_{\mathrm{D}}$ and of $P_{\text {heat }}$ to keep $Z_{\text {eff }}$ at acceptable values. Indeed, when for given $\Gamma_{\mathrm{D}}$ and $P_{\text {heat }}$ the neon seeding rate exceeds a certain threshold, COREDIV simulations indicate that an inward impurity pinch (order $-1 \mathrm{~m} / \mathrm{s}$ ) is triggered (or, alternatively, a decrease in the core impurity diffusivity), esperimentally related to the reduction in the ELM activity and to a significant $Z_{\text {eff }}$ increase. To this point it is worth recalling that the "shot points" at lowest $f_{E L M}$ are experimentally correlated to the highest $\Gamma_{\mathrm{Ne}} / \mathrm{P}_{\mathrm{aux}}$ as well as to the highest energy confinement enhancement factor, $\mathrm{H}_{98}$.

The simplifying assumptions made in COREDIV in order to model self-consistently the complex interaction core-edge plasma certainly attenuate the validity of some quantitative numerical results. However, the simulation of the pulses here considered shows a clear trend and suggests a limit in the level of $\Gamma_{N e} / P_{a u x}$ (hence in $f_{R A D}$ ) at moderate gas puffing rate, if the decrease in ELM activity and the related increase in impurity dwell time is to be avoided. This, of course, refers only to the case of uncontrolled (natural) ELM frequency.

\section{Acknowledgements}

This work has been carried out within the framework of the EUROfusion Consortium and has received funding from the Euratom research and training programme 2014-2018 under grant agreement No 633053. The views and opinions expressed herein do not necessarily reflect those of the European Commission.

This scientific work was financed within the Polish framework of the scientific financial resources allocated for realization of the international co-financed project. 


\section{References}

[1] M. Wischmeier, J. Nucl. Mater. 463 (2015) 22 and 25th IAEA Conference 2014, P. EX/72, St. Petersburg, Russia.

[2] C. Giroud et al., Nucl. Fusion 53 (2013) 113025 and 25th IAEA Conference 2014. EX/P5-25, St. Petersburg, Russia.

[3] T. Puetterich et al., Plasma Phys. Control. Fusion 55 (2013)124036]

[4] A. Huber et al., J. Nucl. Mater. 463 (2015) 445

[5] R. Zagorski, et al., Nucl. Fusion 53 (2013) 073030

[6] G.Telesca et al., Contrib. Plasma Phys. 54, issue 4-6 (2014) 347-352/DOI $10.1002 /$ ctpp. 201410052

[7] G. Telesca et al. J. Nucl. Mater. 463 (2015) 577-581 doi:10.1016/j.jnucmat.2014.11.024

[8] J. Mandrekas and W.M. Stacey, Nucl. Fusion 35 (1995) 843

[9] R. Zagorski, J. Tech. Phys. 37 (1996) 7

[10] Y.Yamamura et al., Report of the IPP Nagoya, IPPJ-AM-26 (1083)

[11] C. Garcia-Rosales et al., J. Nucl. Mater. 218 (1994) 8-17

[12] G. Telesca et al., Plasma Phys. Control. Fusion 53 (2011) 115002

[13] A. Huber et al., 41st EPS Conference on Plasma Physics (2014), P1.031

[14] G. Telesca et al., Nucl. Fusion 40 (2000) 1845

[15] Yu. L. Igitkhanov, Contrib. Plasma Phys. 4/5 (1988) 477

[16] R. Dux et al., J. Nucl. Mater. 390-391(2009) 85

[17] J. Neuhauser et al., Nucl. Fusion 24 (1984) 39

[18] A.E. Jaervinen et al,. Plasma Phys. Control. Fusion 58 (2016) 045011

[19] M. Tokar, «Modeling of localised impulsive injection of neutrals and plasma response » submitted to publication in Plasma Phys. Control. Fusion

[20] S. Brezinsek et al., 57th APS Plasma Physics, Savannah (2015), Vol.60, N. 19, T06.0007

[21] N. den Harder et al., Nucl. Fusion 56 (2016) 026014

[22] A. Loarte et al., Nucl. Fusion 54 (2014) 033007

[23] R. Dux et al., Nucl. Fusion 51 (2011) 053002 


\section{Figure captions}

Fig.1. Experimental time traces of the four pulses examined. The seven time slices of the simulated "shot points" are shown.

Fig.2. Experimental (HRTS) and reconstructed $T_{e}$ and $n_{e}$ profiles for JPN $87194 \mathrm{t}=14.9 \mathrm{~s}$.

Fig.3. a)Tomographic reconstruction of the radiated power density for a typical neon seeded JET pulse at high $P_{\text {heat }}$, JPN $87194 \mathrm{t}=15 \mathrm{~s}$. b) COREDIV simulated radiated power density profile by neon inside the separatrix.

Fig. 4. Comparison experiment-simulation for the seven "shot points" here considered.

Fig. 5. Numerical W fluxes and experimental WI line intensity

Fig.6. a) Numerical pinch velocity vs. experimental ELM frequency. b) Numerical $D_{\text {imp }} / D_{\text {main }}$ in the core plasma vs. experimental ELM frequency

Fig.7. Experimental $\Gamma_{N e} / P_{\text {heat }}$ vs $\Gamma_{\mathrm{D}}$ with different symbols for $f_{E L M}>40 \mathrm{~Hz}$ and $f_{E L M}<20 \mathrm{~Hz}$.

Fig. 8. Numerical Ne fluxes versus input Ne recycling coefficient for JPN 87191.

Fig.9. $P_{\text {rad }}^{T O T}, P_{\text {rad }}^{S O L} / P_{\text {rad }}^{T O T}$ and $Z_{\text {eff }}$ as a function of the ratio between input $\Gamma_{\mathrm{Ne}}$ and $\Gamma_{\mathrm{Ne}}$ of pulse 87191. 

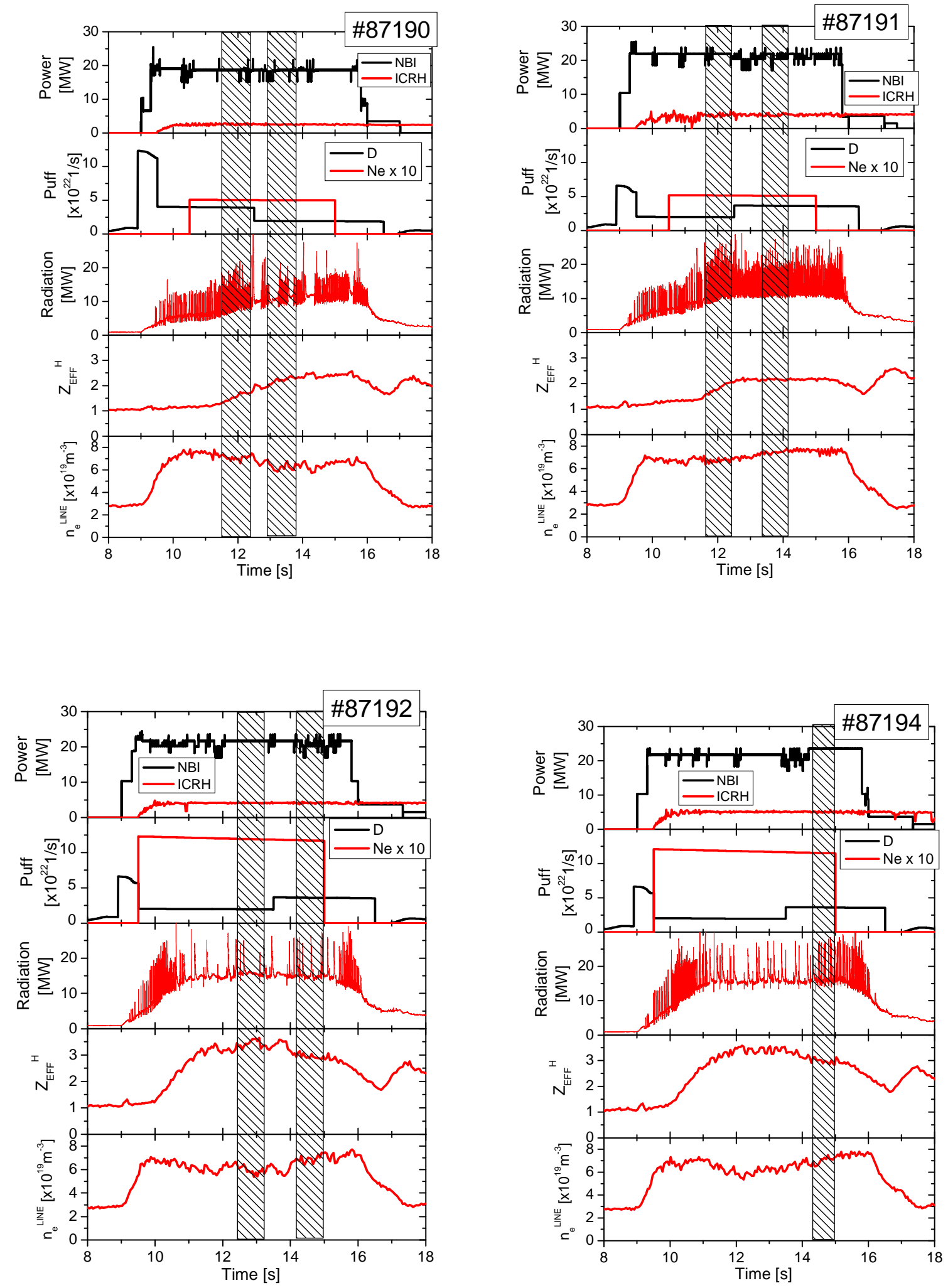

Fig. 1 


\begin{tabular}{|c|c|c|c|c|c|c|c|c|c|c|}
\hline $\begin{array}{l}P_{\text {heat }} \\
{[\mathrm{MW}]}\end{array}$ & $\begin{array}{c}\Gamma_{D} \\
{\left[\times 10^{22} 1 / s\right]}\end{array}$ & $\begin{array}{c}\Gamma_{\mathrm{Ne}} \\
{\left[\times 10^{21} \mathrm{el} / \mathrm{s}\right]}\end{array}$ & $\begin{array}{c}\mathrm{n}_{\mathrm{e}}(0) \\
{\left[\times 10^{19} \mathrm{~m}^{-3}\right]}\end{array}$ & $\begin{array}{l}T_{e}(0) \\
k e V\end{array}$ & $\mathrm{H}_{98}$ & $f_{\text {RAD }}$ & $\begin{array}{l}P_{\text {RAD }} \\
{[\mathrm{MW}]}\end{array}$ & $\begin{array}{c}Z_{\text {EFF }} \\
\text { brem. }\end{array}$ & $\mathrm{P}_{\text {DIV }} / \mathrm{P}_{\text {TOT }}$ & $\begin{array}{l}f_{E L M} \\
{[H z]}\end{array}$ \\
\hline \multirow[t]{2}{*}{22} & 3.6 & 5 & 8.3 & 3.5 & 0.68 & 0.47 & 10.4 & 1.9 & 0.42 & 40 \\
\hline & 1.9 & 5 & 7.6 & 4.4 & 0.76 & 0.52 & 11.5 & 2.4 & 0.43 & $40 / 2$ \\
\hline \multirow[t]{2}{*}{26.5} & 3.6 & 5 & 8.4 & 4.1 & 0.73 & 0.54 & 14.3 & 2.1 & 0.4 & 65 \\
\hline & 1.9 & 5 & 8.0 & 4.4 & 0.68 & 0.47 & 12.3 & 2.0 & 0.35 & 60 \\
\hline \multirow[t]{2}{*}{26.5} & 3.6 & 12 & 8.0 & 5.3 & 0.79 & 0.57 & 15.2 & 3.0 & 0.39 & 9 \\
\hline & 1.9 & 12 & 7.5 & 5.5 & 0.79 & 0.54 & 14.3 & 3.3 & 0.4 & 5 \\
\hline 29.5 & 3.6 & 12 & 8.0 & 5.7 & 0.8 & 0.6 & 18.1 & 2.9 & 0.4 & 15 \\
\hline
\end{tabular}

Table I. Summary of the experimental data 


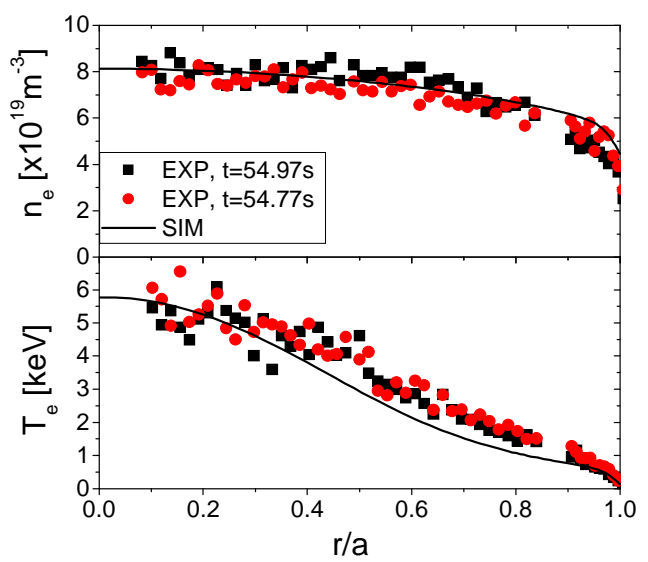

Fig. 2 


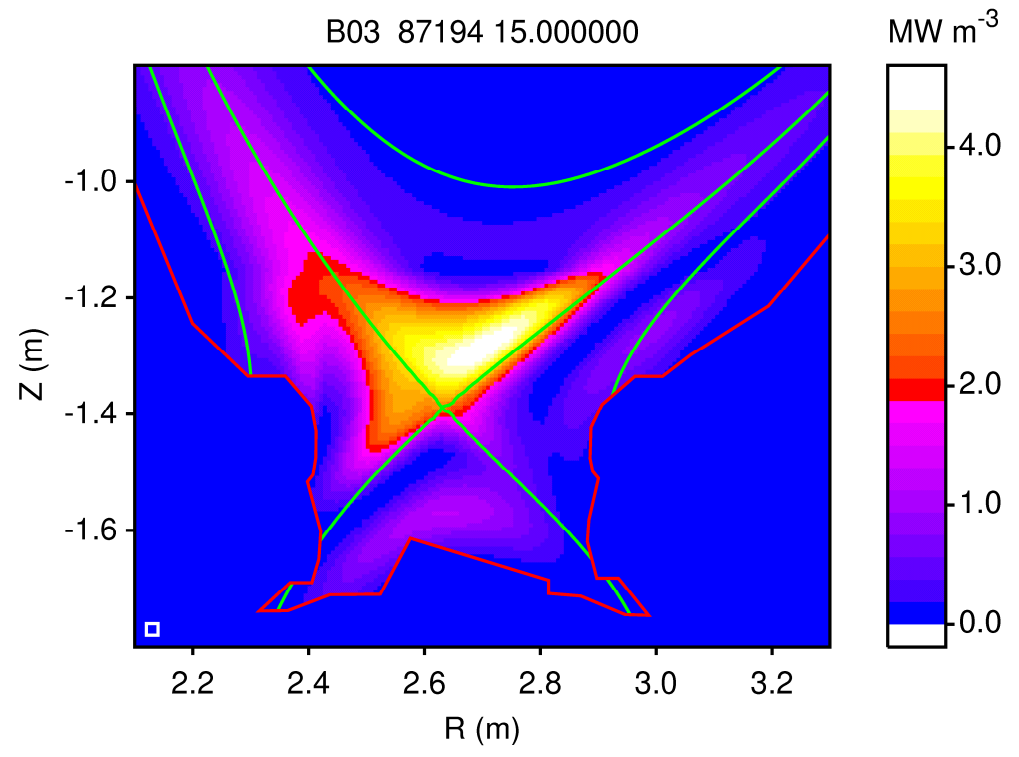

a)

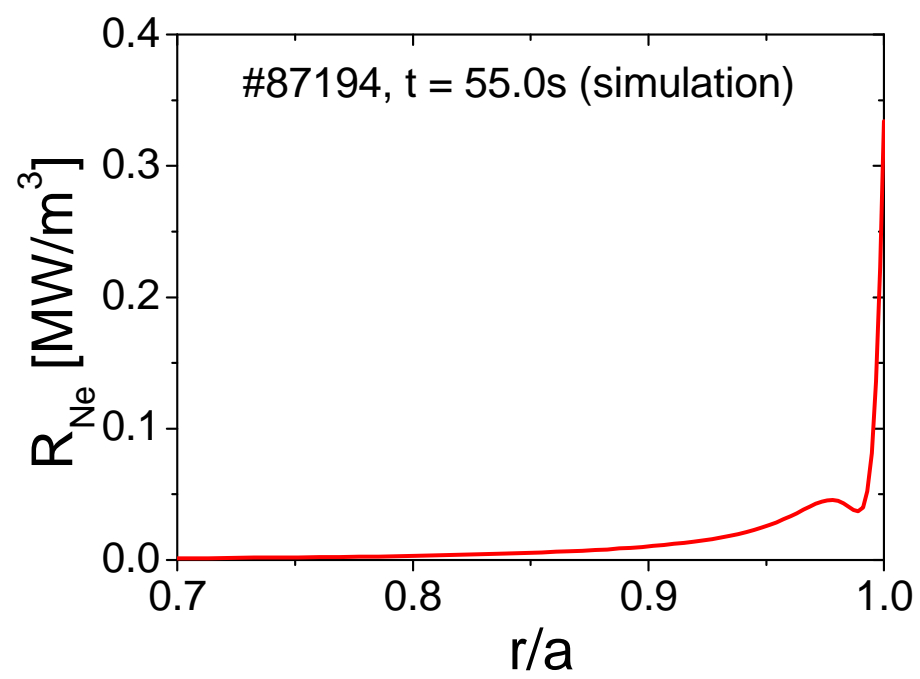

b)

Fig. 3 

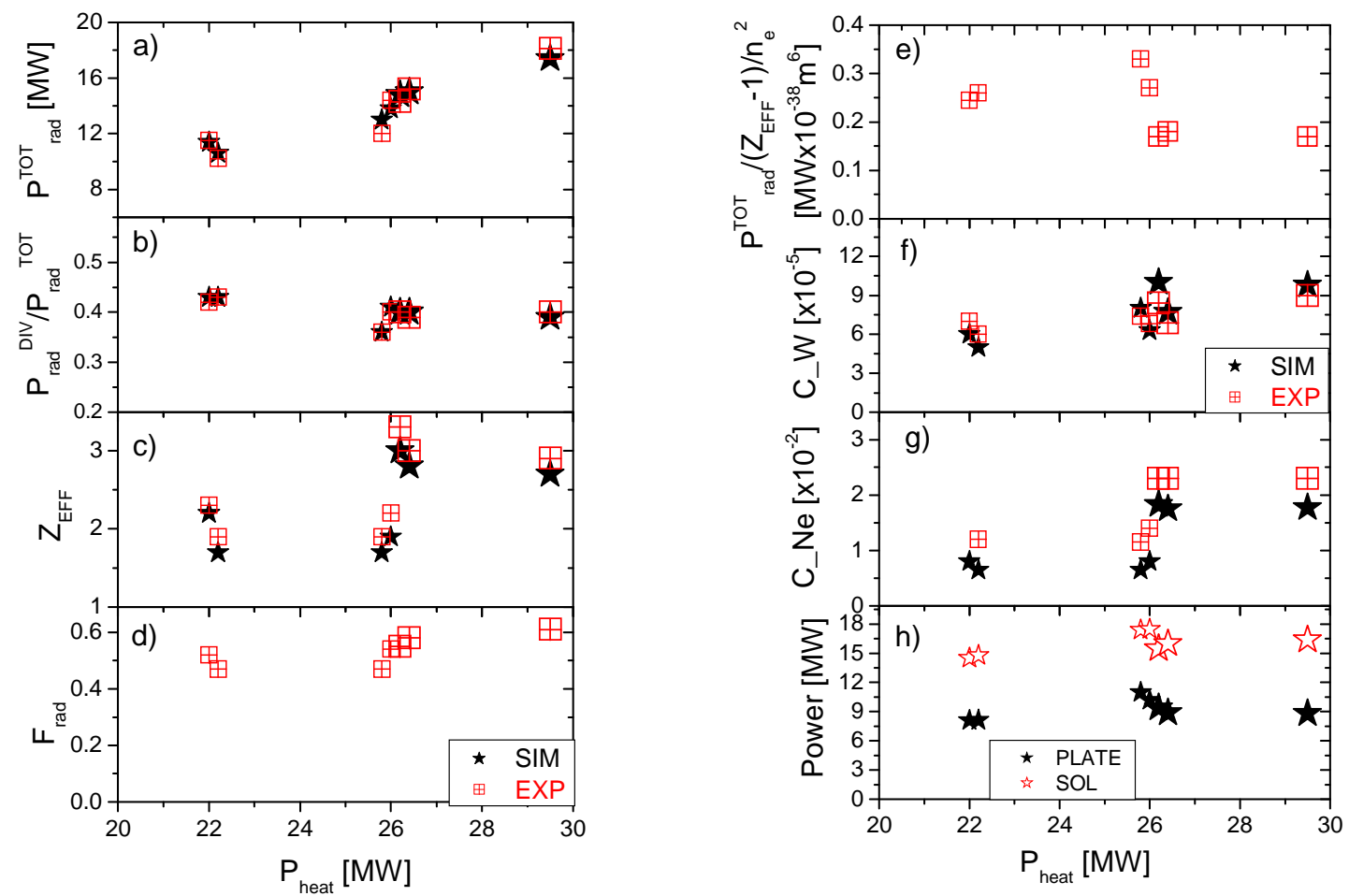

Fig. 4 


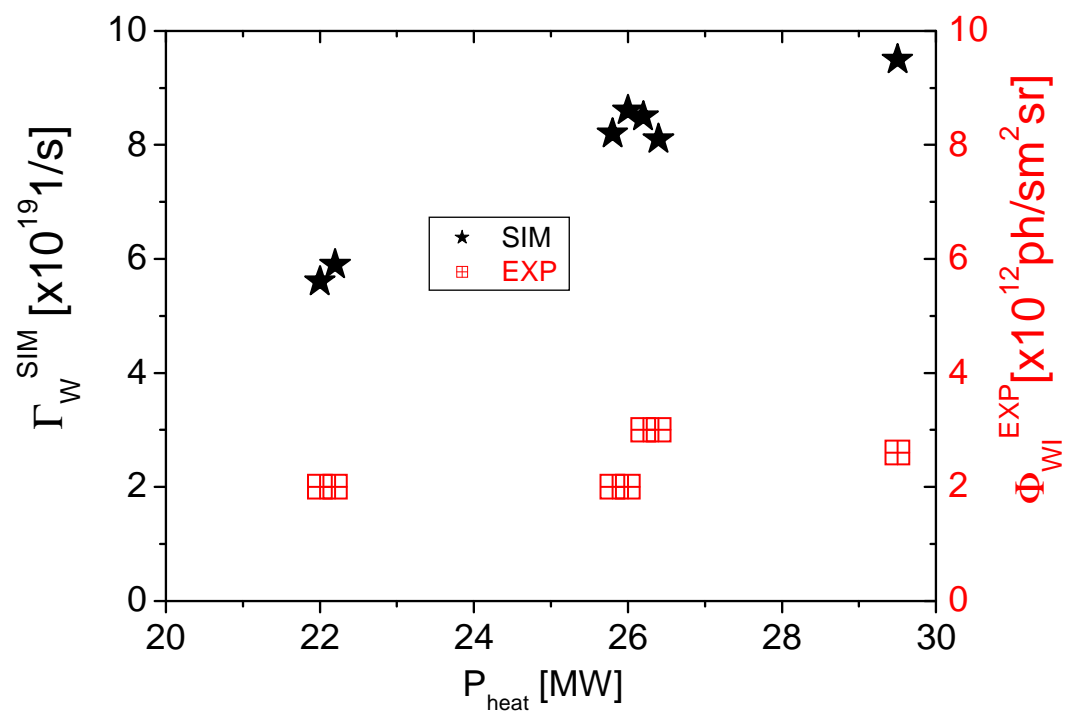

Fig. 5 


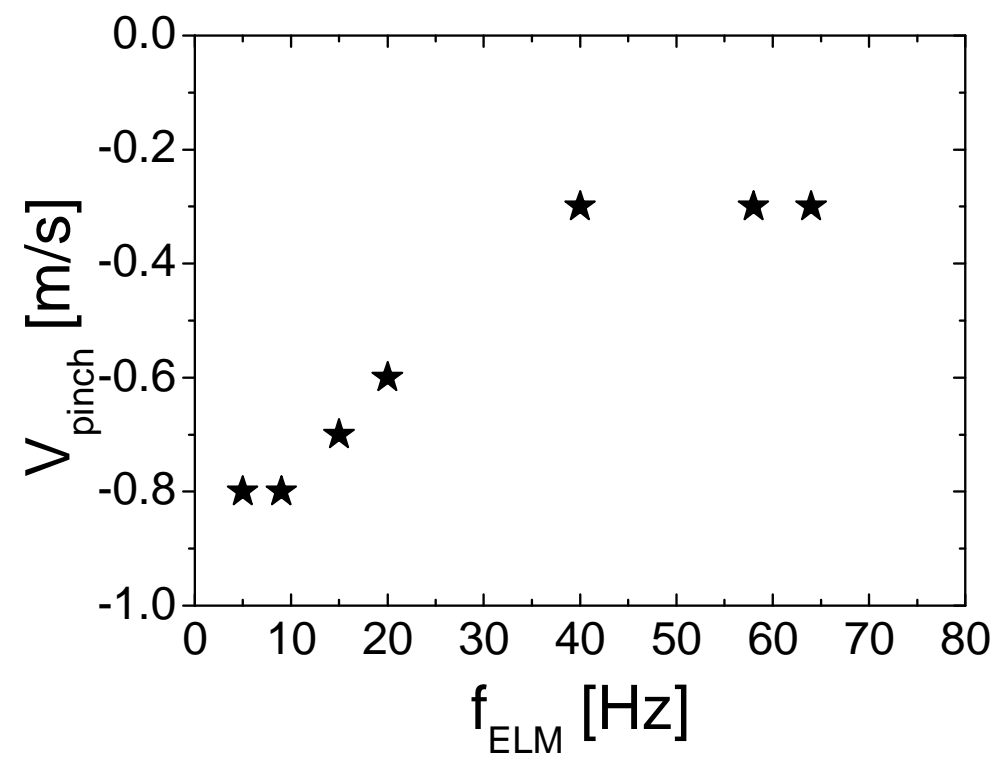

Fig. 6a

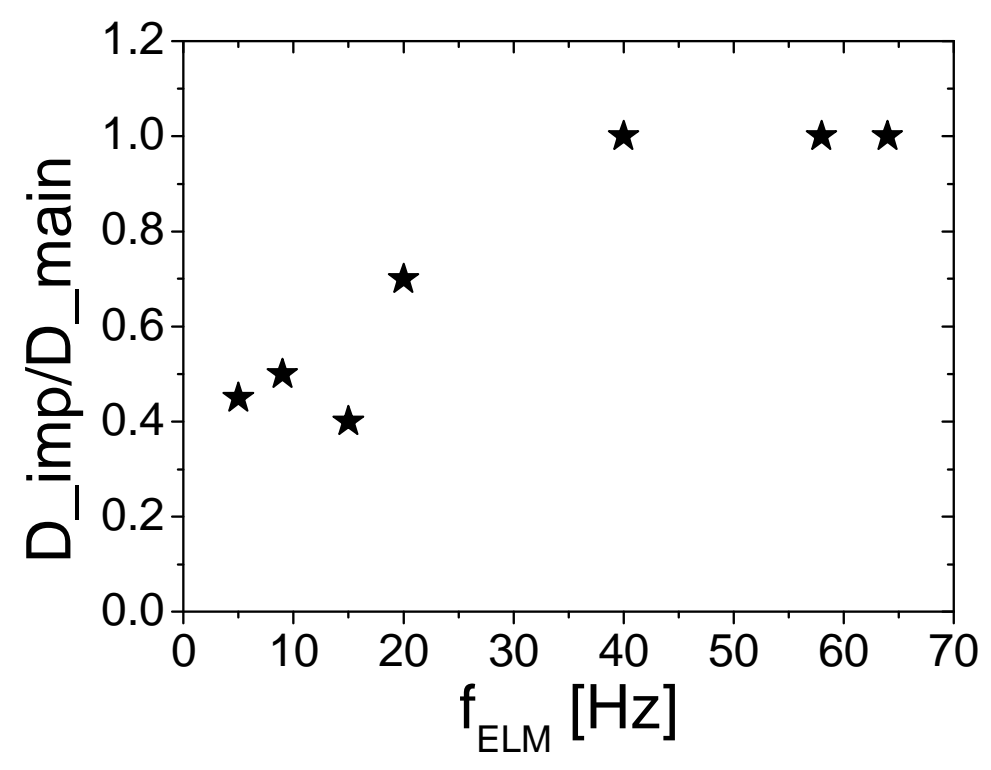

Fig. $6 b$ 


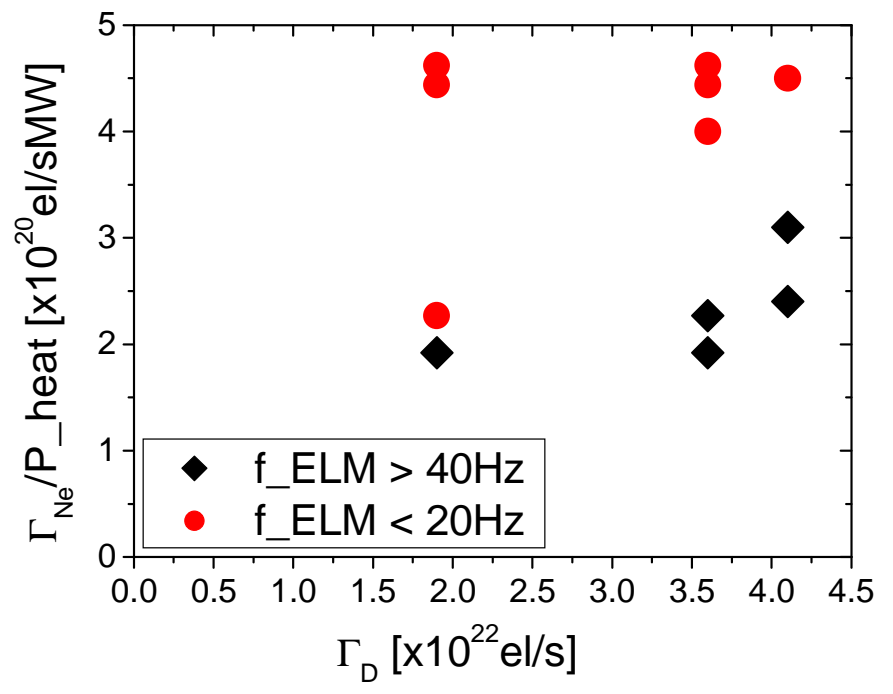

Fig. 7 


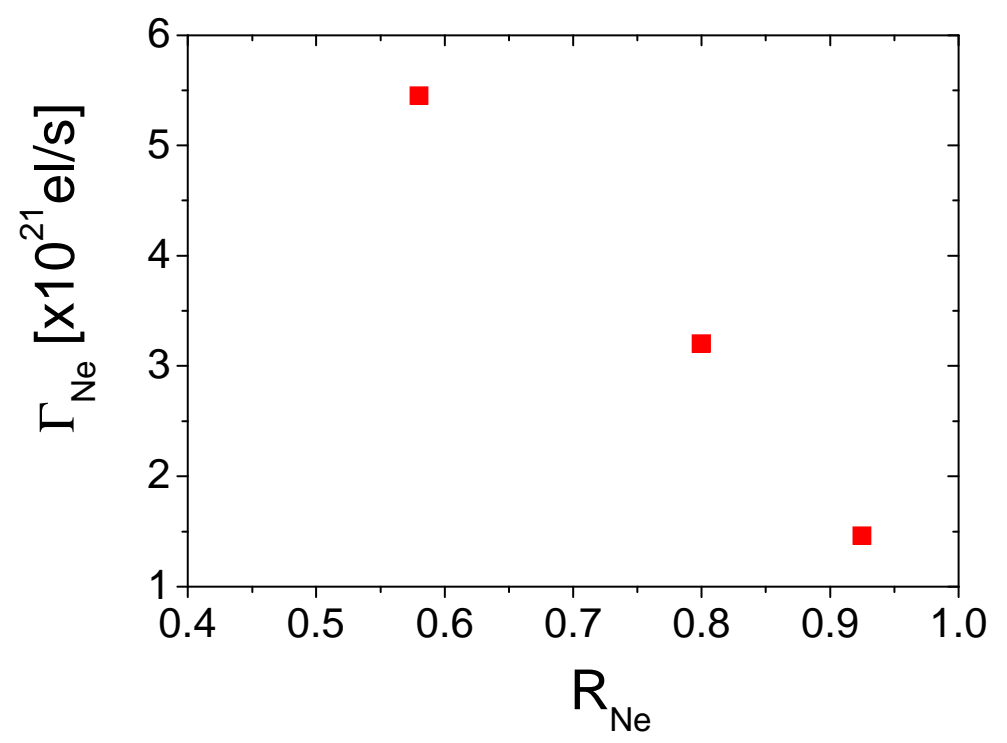

Fig 8 


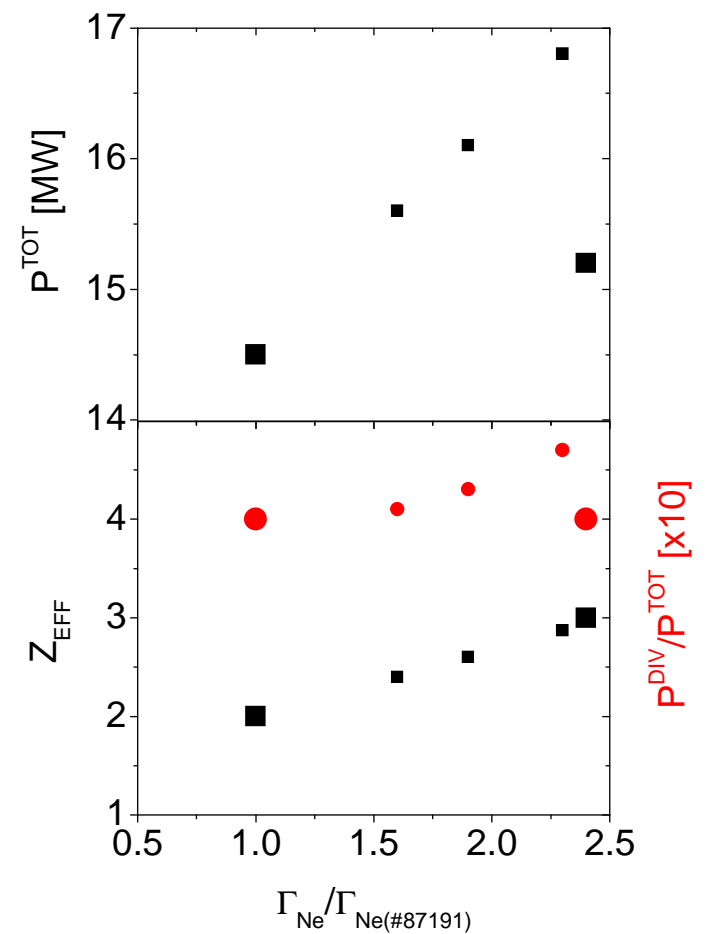

Fig.9 